\title{
Aislamiento de Leishmania infantum a partir de Canis familiaris en área urbana del Caribe colombiano
}

\author{
ISOLATION OF Leishmania infantum FROM Canis familiaris IN AN URBAN AREA \\ of THe Colombian Caribbean
}

\author{
Matilde Elena Rivero-Rodríguez ${ }^{1,2}$, Jorge Luis Rodríguez-Jiménez ${ }^{1}$, \\ Alveiro Pérez-Doria ${ }^{1}$, Eduar Elías Bejarano ${ }^{1}$
}

\section{Resumen}

\begin{abstract}
Se registra el aislamiento de parásitos del género Leishmania a partir de una biopsia de ganglio poplíteo obtenida de un perro con leishmaniasis visceral canina. El animal procedía del área urbana del municipio de Ovejas, Sucre, Colombia. La prueba de Inmunofluorescencia Indirecta fue positiva para anticuerpos anti-Leishmania spp hasta una dilución de 1/128. La tipificación de $L$. infantum fue realizada por electroforesis de isoenzimas y por análisis de la secuencia del gen citocromo b y el gen nuclear que codifica para la proteína de choque térmico de $70 \mathrm{Kda}(\mathrm{Hsp} 70)$. El presente hallazgo constituye la primera confirmación de infección por $L$. infantum en caninos en área urbana del Caribe colombiano.
\end{abstract}

Palabras clave: L. infantum; perro; leishmaniasis visceral canina; IFI; isoenzimas; PCR

\section{Abstract}

The isolation of parasites of the genus Leishmania is reported from a popliteal ganglion biopsy obtained from a dog with canine visceral leishmaniasis. The dog lived in the urban area of the municipality of Ovejas, Sucre, Colombia. The Indirect Immunofluorescence test was positive for anti-Leishmania spp antibodies up to a dilution of $1 / 128$. The typing of $L$. infantum was performed by isoenzyme electrophoresis and by

\footnotetext{
${ }^{1}$ Investigaciones Biomédicas, Universidad de Sucre, Colombia

${ }^{2}$ E-mail: matyrivero2010@gmail.com
}

Recibido: 15 de octubre de 2017

Aceptado para publicación: 30 de abril de 2018 
analysis of the sequence of the cytochrome $b$ gene and the nuclear gene that codes for the heat shock protein of $70 \mathrm{Kda}$ (Hsp70). The present finding is the first confirmation of infection by $L$. infantum in canines in the urban area of the Colombian Caribbean.

Key words: L. infantum; dog; leishmaniasis; IFAT; isoenzymes; PCR

\section{INTRODUCCCIÓN}

La leishmaniasis es una parasitosis causada por protozoarios del género Leishmania que afecta la piel, mucosas y órganos internos (Alvar et al., 2012). En Colombia se han presentado más de 10000 casos anuales en personas entre 2005 y 2014, principalmente en áreas rurales, representando un problema de Salud Pública. Dentro de las formas clínicas, la leishmaniasis visceral (LV), causada por Leishmania infantum, puede ser fatal si no se diagnostica y se trata a tiempo.

Dentro del ciclo zoonótico de la LV, los parásitos del género Leishmania son transmitidos por insectos flebotomíneos del género Lutzomyia. Los patrones de distribución en casos humanos no solo dependen de la distribución de los vectores, sino también de animales silvestres y domésticos que actúan como reservorios de los parásitos y que constituyen la fuente de infección para el insecto vector. Como posibles reservorios silvestres de L. infantum en Colombia se ha señalado a la rata espinosa de Colombia (Proechimys canicollis) y la zarigüeya (Didelphys marsupialis) (Travi et al., 1994, 1998). Además, existe evidencia experimental y epidemiológica para considerar al perro (Canis familiaris) como el principal reservorio doméstico y peri-doméstico (Ashford, 1996).

En caninos infectados con Leishmania spp se puede presentar una infección subclínica, una enfermedad autolimitante o una enfermedad severa que no se puede controlar (Baneth y Aroch, 2008). Se reconoce que cerca de la mitad de los perros infectados por L. infantum presenta signos de la enfermedad, pudiendo desarrollar una enfermedad fatal conocida como leishmaniasis visceral canina (LVC), caracterizada por linfoadenomegalia, atrofia muscular, úlceras en piel, pérdida de apetito y de peso, onicogrifosis, esplenomegalia, poliuria y polidipsia, entre otros signos clínicos (DantasTorres, 2009).

El diagnóstico preciso y temprano, así como el oportuno tratamiento de LVC son factores importantes en salud pública y veterinaria. El diagnóstico diferencial es necesario, debido a que los perros pueden infectarse con varias especies de tripanosomátidos y producir reacción cruzada en las pruebas serológicas (Solano-Gallego et al., 2014). El diagnóstico de LVC se hace mediante pruebas serológicas, parasitológicas y, en las últimas décadas, por pruebas moleculares ((Travi et al., 2018).

En el municipio de Ovejas del departamento de Sucre, Colombia, se encuentra un foco mixto de leishmaniasis visceral y cutánea, donde se registran cerca de 50 casos anuales en humanos de leishmaniasis (92\% de LC y $6 \%$ de LV) (INS, sf). Además, en los últimos años, se han presentado casos en el área urbana del municipio (INS, 2013).

Teniendo en cuenta la circulación de varias especies de tripanosomátidos en la región, el papel de $C$. familiaris en el mantenimiento del ciclo epidemiológico de parásitos causantes de leishmaniasis visceral y la falta de especificidad de los métodos serológicos empleados para la vigilancia y diagnóstico de 
la LVC, se describe en el presente trabajo el aislamiento de una cepa de L. infantum a partir de una biopsia del ganglio poplíteo de un canino que presentaba signos compatibles con LVC, residente en área urbana del Caribe colombiano, donde se registran casos humanos de leishmaniasis visceral.

\section{Materiales y Métodos}

\section{Caso Clínico}

El caso clínico fue reportado en el municipio de Ovejas, departamento de Sucre, en el Caribe colombiano, ubicado a una altitud de $265 \mathrm{~m}$. Durante el desarrollo de actividades de vigilancia epidemiológica de LVC llevadas a cabo en el barrio Plaza de la Cruz de este municipio, se encontró un perro con signos clínicos compatibles con esta enfermedad. Se visitó el domicilio de los propietarios del canino, una vivienda construida con materiales prefabricados, la cual tenía una zona de labores tipo caney (techo construido en madera y palmas, piso prefabricado), ubicada en el patio a menos de $10 \mathrm{~m}$ de distancia de un remanente de bosque.

El perro de cuatro años era macho y mestizo. La propietaria refirió que la mayoría de los signos clínicos empezaron aproximadamente 45 días antes de la visita. Se obtuvo el consentimiento informado de los propietarios y se procedió a la realización de un examen físico y a la toma de una muestra de sangre de $5 \mathrm{ml}$ de la vena cefálica, la cual se centrifugó a $3000 \mathrm{rpm}$ durante 10 minutos para la obtención del suero. Además, mediante el procedimiento de 'Biopsia Aspirativa con Aguja Fina (BACAF)', se tomó una biopsia del ganglio poplíteo. La biopsia fue incluida en $200 \mu 1$ de medio RPMI 1640 (Ref. R6504, Sigma) y colocada directamente en un tubo con medio Novy-moneal-Nicolle (NNN, Bacto-agar, cloruro de sodio, suplementado con sangre de conejo).

\section{Pruebas Realizadas}

Las muestras de suero sanguíneo fueron analizadas mediante la técnica de Inmunofluorescencia Indirecta (IFI), según el protocolo de Camargo (1996). Se utilizaron promastigotes de L. infantum de una cepa local (MHOM/CO/2013/133) como antígenos y anti-IgG de perro marcada con fluoresceína como conjugado (Sigma). La interpretación de los resultados de IFI se hizo según el protocolo de diagnóstico recomendado por el Instituto Nacional de Salud de Colombia (INS, 2010), donde se considera positivo a lecturas de fluorescencia en suero a una dilución $>1 / 32$.

Se aisló una cepa de Leishmania de la muestra de ganglio poplíteo en medio NNN y propagada en medio RPMI 1640. La cepa fue identificada usando la técnica estándar de tipificación por Electroforesis de Enzimas Multilocus (MLEE) en acetato de celulosa. Se evaluaron los perfiles electroforéticos de la isoenzima fosfoglucosa mutasa (PGM), glucosa 6 fosfato deshidrogenasa (G6PDH), fosfogluconato deshidrogenasa (6PGDH), nucleósido hidrolasa $(\mathrm{NH})$, superóxido dismutasa (SOD), glucosa fosfato isomerasa (GPI) y manosa fosfato isomerasa (MPI).

Además, se realizó detección molecular basada en la secuencia del gen mitocondrial Citocromo b (cytb) y del gen nuclear que codifica para la proteína de choque térmico de 70 kda (Hsp70). La extracción del ADN de los parásitos fue realizada con el kit comercial QIAamp DNA Mini Kit. La PCR anidada del gen cytb fue realizada con los cebadores LCBF1 y LCBR2 (Luyo-Acero et al., 2003), que delimitan un segmento de aproximadamente $860 \mathrm{pb}$ del gen cytb, y en la segunda ronda se usaron los cebadores $\mathrm{CB}$ Cytb-F y CB-Cytb-B (Schmid-Hempel y Tognazzo, 2010), que flanquean un fragmento de $750 \mathrm{pb}$ de este mismo gen. Como control positivo se utilizó ADN de una cepa de $L$. infantum (MHOM/CO/2013/133) y como 
control negativo mezcla de reacción sin ADN y ADN de caninos no infectados. Adicionalmente, se amplificó el gen Hsp70 con los cebadores Hsp70 Sen y Hsp70 Ant (Fraga et al., 2010), que delimitan un fragmento de aproximadamente $1460 \mathrm{pb}$. En el proceso se usaron los controles antes descritos. La secuenciación se realizó en un equipo ABI 3130XL de electroforesis capilar. Los electroforegramas obtenidos fueron editados de forma automatizada en el programan Genious 9.1.2 ${ }^{\circledR}$ y fueron revisados de forma manual para generar una secuencia consenso. Con el programa MAFFT v. 7.222 se alinearon las secuencias obtenidas con secuencias homólogas de cepas de referencia de la Organización Mundial de la Salud, consignadas en GenBank. Con base en el alineamiento obtenido, se estimó el modelo de sustitución que mejor explica la diversidad observada entre las secuencias y se realizaron las reconstrucciones filogenéticas en el programa Mega 7.

\section{Resultados}

En la valoración física, el canino presentó caquexia, onicogrifosis, infección ocular, lesiones cutáneas (Figura 1), hematuria y mal estado general, signos clínicos que en conjunto son compatibles con LVC. El paciente canino falleció tres días después de la toma de muestras, de allí que no se pudo realizar ensayos de infectividad para los vectores. El diagnóstico serológico fue positivo para la presencia de IgG anti-Leishmania en diluciones de 1/32, 1/64 y 1/128. Las siete enzimas evaluadas por MLEE mostraron un perfil isoenzimático compatible con la cepa de referencia de L. chagasi, MHOM/BR/ 2013/PP75, sinónimo junior de L. infantum.

Como resultado de la PCR del gen Cyt $\mathrm{B}$, se amplificó la banda esperada. Después de hacer la edición de los electroforegramas, se obtuvo una secuencia consenso de 711 bases de longitud y fue depositada en

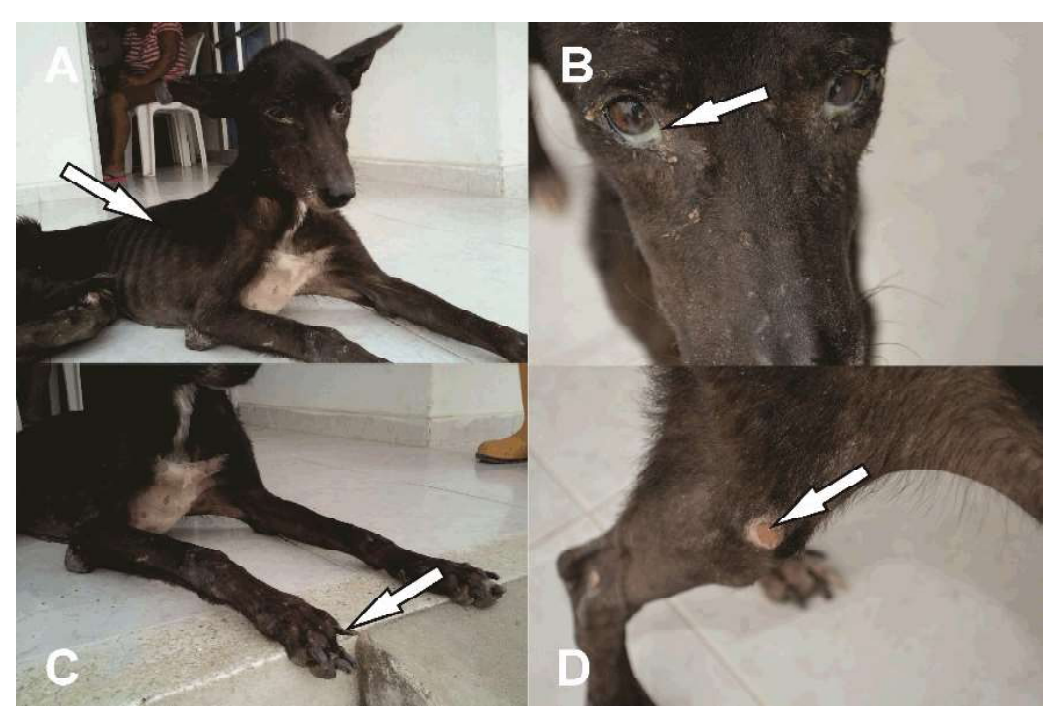

Figura 1. Signos clínicos presentes en el canino. A. Caquexia. B. Infección ocular. C. Onicogrifosis. D. Lesión cutánea 




Figura 2. Árbol filogenético basado en 21 secuencias nucleotídicas del gen Citocromo b de especies del género Leishmania. El análisis filogenético se realizó por el método de Máxima Verosimilitud, previo ajuste de las distancias genéticas con el modelo de Hasegawa-Kishino-Yano (HKY). En la base de cada clado, se muestra el soporte de rama, expresado como el porcentaje de veces que el análisis produjo la misma asociación entre las secuencias (10 000 seudoréplicas). Para cada taxón se indica el número de acceso en GenBank y el nombre de la especie. En el recuadro negro se observa la secuencia KX443560 obtenida de los parásitos aislados del canino

Genbank (número de acceso KX443560). Así mismo, se amplificó y secuenció un fragmento de $1460 \mathrm{pb}$ del gen Hsp70, obteniéndose una secuencia consenso de $1372 \mathrm{pb}$ que fue registrada en GenBank (número de acceso KX759517). La búsqueda de similitud con BLAST indicó que tanto la secuencia del gen
Cyt B como la secuencia del gen Hsp70 corresponden a $L$. infantum. En el caso del gen Cyt b con valores de $99 \%$ de cobertura, $99 \%$ de identidad, con un valor $\mathrm{E}$ de 0.0 respecto a la secuencia de $L$. infantum de referencia con el número de acceso HQ908261.1. En el caso del gen hsp 70 se obtuvo un valor de $98 \%$ 
de cobertura, $99 \%$ de identidad y un valor $\mathrm{E}$ de 0.0 respecto a la secuencia con el número de acceso FN395031.1.

En las reconstrucciones filogenéticas realizadas para cada marcador molecular con el método de Máxima Verosimilitud (EMV), ajustado con el modelo de evolución de Hasegawa-Kishino-Yano (HKY) (Hasegawa et al., 1985), en la topología del árbol, las secuencias nucleotídicas derivadas de los parásitos del canino (cytb y HSP70) se ubicaron dentro del clado que agrupa a las especies del complejo L. donovani, con altos valores de soporte de rama (100\%). Las secuencias de interés agruparon con secuencias de L. infantum, especie del complejo presente en América, y fue independiente de los métodos evaluados y del marcador usado en la reconstrucción; sin embargo, solo se muestra el análisis filogenético de las secuencias nucleotídicas del gen Cyt B, analizadas por EMV (Figura 2). Teniendo en cuenta estos resultados y las distancias genéticas intraespecíficas obtenidas entre este par de especies $(0.426 \%$ con las cepas EF579895 y EF579897), se infiere que la cepa aislada del canino corresponde a L. infantum, hecho que es consistente con los resultados del perfil isoenzimático obtenido por MLEE y demuestra la equivalencia de estos métodos de identificación, para este caso particular.

\section{Discusión}

Se reconocen dos escenarios en leishmaniasis canina: en el caso de L. infantum, los caninos son considerados como buenos reservorios del parásito, pudiendo sufrir la enfermedad (LVC), mientras que en el caso de parásitos del subgénero Viannia, si bien se han encontrado caninos con infección natural por estas especies, podrían no ser buenos reservorios (Vélez et al., 2012); sin embargo, teniendo en cuenta el carácter focal de esta parasitosis, es importante, estudiar cada foco.
En el caso de focos mixtos, como ocurre en el municipio de Ovejas, con casos de LC y LV, donde además se han encontrado caninos infectados con L. guyanensis y $L$. braziliensis (Rivero-Rodríguez et al., 2015) es importante el diagnóstico diferencial para evitar el sacrificio innecesario de caninos infectados. En este caso, la presencia de signos clínicos y títulos elevados de anticuerpos como respuesta a la infección serían suficientes para el diagnóstico de LVC; sin embargo, esta situación no siempre se presenta en estudios epidemiológicos, donde se observan animales sin signos clínicos y resultados no concluyentes de IFI, ya que se pueden presentar reacciones cruzadas con otros tripanosomátidos (Solano-Gallego et al., 2014). Es así que la identificación de la especie sería determinante para no desestimar o sobreestimar el papel que desempeñan las poblaciones de caninos en el mantenimiento del ciclo epidemiológico de especies causantes de leishmaniasis.

En este sentido, aunque la técnica MLEE es más laboriosa y requiere del previo aislamiento de la cepa, sigue siendo la técnica de referencia para la clasificación taxonómica de especies del género Leishmania (OMS, 2010) y confirmar la presencia de parásitos viables. En el presente caso, se logró establecer la identificación de L. infantum posterior al aislamiento, a través del análisis isoenzimático y de los genes Citocromo b y Hsp70.

El hallazgo de L. infantum en un canino del área urbana del municipio de Ovejas coincide con la epidemiología de la leishmaniasis visceral en Colombia, si se tiene en cuenta que en la costa norte de Colombia, así como en casi todo el territorio nacional, se ha descrito que estos animales pueden actuar como reservorios domésticos de los parásitos causantes de LV en humanos ubicados en zonas rurales y urbanas del territorio nacional (Travi et al., 1998; Santaella et al., 2011). 
Es pertinente resaltar que, a pesar de que el municipio de Ovejas, en los Montes de María, representa uno de los focos de LV más importantes de la costa norte de Colombia y del país, es la primera vez que se logra el aislamiento de parásitos del género Leishmania a partir de una muestra de canino en este municipio y en un área urbana del Caribe colombiano. Los resultados podrían indicar que los habitantes del área urbana del municipio conviven con factores de riesgo, especialmente la población infantil que es la más afectada por esta parasitosis (OMS, 2010).

\section{Agradecimientos}

Este trabajo fue realizado gracias a la financiación de Departamento Administrativo de Ciencia, Tecnología e Innovación (COLCIENCIAS), código 112956934732 , Colombia, convocatoria 569.

\section{Literatura Citada}

1. Alvar J, Velez ID, Bern C, Herrero M, Desjeux P, Cano J, Jannin J, et al. 2012. Leishmaniasis worldwide and global estimates of its incidence. Plos One 7(5). doi: 10.1371/journal.pone. 0035671

2. Ashford RW. 1996. Leishmaniasis reservoirs and their significance in control. Clin Dermatol 14: 523-532. doi: 10.1016/0738-081X(96)00041-7

3. Baneth G, Aroch I. 2008. Canine leishmaniasis: a diagnostic and clinical challenge. Vet J 175: 14-15. doi: 10.1016/ j.tvj1.2006.11.011

4. Camargo M. 1966. Fluorescent antibody test for the serodiagnoses of American trypanosomiasis. Technical modification employing preserved culture forms of Trypanosoma cruzi in a slide test. Rev Inst Med Trop Sao Paulo 8: 227-234.
5. Dantas-Torres F. 2009. Canine leishmaniosis in South America. Parasit Vectors 2 (Suppl 1): S1. doi: 10.1186/ 1756-3305-2-S1-S1

6. Fraga J, Montalvo AM, De Doncker $S$, Dujardin JC, Van der Auwera G. 2010. Phylogeny of Leishmania species based on the heat-shock protein 70 gene. Infect Genet Evol 10: 238-245. doi: 10.1016/j.meegid.2009.11.007

7. Hasegawa M, Kishino H, Yano T. 1985. Dating of the human-ape splitting by a molecular clock of mitochondrial DNA. J Mol Evol 22: 160-174.

8. [INS] Instituto Nacional de Salud. sf. [Internet]. Disponible en: https:// www.ins.gov.co/buscador-eventos/Paginas/Vista-Boletin-Epidemilogico.aspx

9. [INS/ Instituto Nacional de Salud. 2014. Protocolo de vigilancia en salud pública. Leishmaniasis. Colombia. [Internet]. Disponible en: http://www.clinicamedi-help.com/documentos/protocolos/PRO\%20Leishmaniasis.pdf

10. Luyo-Acero GE, Uezato H, Oshiro M, Takei K, Kariya K, Katakura K, Gomez-Landires E, Nonaka S. 2003. Sequence variation of the Cytochrome $b$ gene of various human infecting members of the genus Leishmania and their phylogeny. Parasitology 128: 483-491.

11. [OMS] Organizacion Mundial de la Salud. 2010. Control de la leishmaniasis. Informe de una reunión del Comité de Expertos de la OMS sobre el Control de las Leishmaniasis. Ginebra. [Internet]. Disponible en: http://apps.who.int/iris/ bitstream/handle/10665/82766/ WHO_TRS_949_spa.pdf;jses sionid=89DE7A 1 - 0 F 1 F 6 EA 0A1FC141BED988D430? Sequence $=1$

12. Rivero-Rodríguez ME, Rodríguez J, Meza E, Pérez-Doria A, Bejarano E. 2015. Primer hallazgo de Leishmania guyanensis y Leishmania braziliensis en perros asintomáticos del foco urbano de leishmaniasis del municipio de Ovejas, Sucre. Biomédica 35 (Supl 4): 113 (Resumen). 
13. Santaella J, Ocampo CB, Saravia NG, Mendez F, Gongora R, Gomez MA, Munstermann LE, Quinnell RJ. 2011. Leishmania (Viannia) infection in the domestic dog in Chaparral, Colombia. Am J Trop Med Hyg 84: 674-680. doi: 10.4269/ajtmh.2011.10-0159

14. Schmid-Hempel R, Tognazzo M. 2010. Molecular divergence defines two distinct lineages of Crithidia bombi (Trypanosomatidae), parasites of bumblebees. J Eukaryot Microbiol 57: 337-345. doi: $10.1111 / \mathrm{j} .1550$ 7408.2010.00480.x

15. Solano-Gallego L, Villanueva-Saz, $S$, Carbonell M, Trotta M, Furlanello T, Natale A. 2014. Serological diagnosis of canine leishmaniosis comparison of three commercial ELISA tests (Leiscan, ID Screen and Leishmania 96), a rapid test (Speed Leish K) and an in-house IFAT. Parasit Vectors 7: 111. doi: 10.1186/ 1756-3305-7-111

16. Travi BL, Jaramillo C, Montoya J, Segura I, Zea A, Goncalves A, Velez ID. 1994. Didelphis marsupialis, an important reservoir of Trypanosoma
(Schizotrypanum) cruzi and Leishmania (Leishmania) chagasi in Colombia Am. J. Trop. Med. Hyg 50: 557-565. doi: 10.4269/ajtmh.1994.50.557

17. Travi BL, Osorio Y, Becerra MT, Adler GH. 1998. Dynamics of Leishmania chagasi infection in small mammals of the undisturbed and degraded tropical dry forests of northern Colombia. Trans R Soc Trop Med Hyg 92: 275-278. doi: https://dx.doi.org/ 10.1016/S0035-9203(98)91009-4

18. Travi BL, Cordeiro-da-Silva A, Dantas-Torres F, Miro G 2018. Canine visceral leishmaniasis: diagnosis and management of the reservoir living among us. Plos Negl Trop Dis 12(1): e0006082. doi: 10.1371/journal.pntd.0006082

19. Velez ID, Carrillo LM, Lopez L, Rodriguez E, Robledo SM. 2012. An epidemic outbreak of canine cutaneous leishmaniasis in Colombia caused by Leishmania braziliensis and Leishmania panamensis. Am J Trop Med Hyg 86: 807-811. doi: 10.4269/ ajtmh. 2012.11-0408 